\title{
Enhancing GABA Signaling during Middle Adulthood Prevents Age-Dependent GABAergic Interneuron Decline and Learning and Memory Deficits in ApoE4 Mice
}

\author{
Leslie M. Tong, ${ }^{1,2}$ Seo Yeon Yoon, ${ }^{1}$ Yaisa Andrews-Zwilling, ${ }^{1}{ }^{\oplus}$ Alyssa Yang, ${ }^{1}$ Victoria Lin, ${ }^{1}$ Hanci Lei, ${ }^{1}$ \\ and Yadong Huang ${ }^{1,2,3,4}$ \\ ${ }^{1}$ Gladstone Institute of Neurological Disease, San Francisco, California 94158, and ${ }^{2}$ Biomedical Sciences Graduate Program, and Departments of ${ }^{3}$ Neurology \\ and ${ }^{4}$ Pathology, University of California, San Francisco, California 94143
}

\begin{abstract}
Apolipoprotein E4 (apoE4) is the major genetic risk factor for Alzheimer's disease (AD). However, the underlying mechanisms are still poorly understood. We previously reported that female apoE4 knock-in (KI) mice had an age-dependent decline in hilar GABAergic interneurons that correlated with the extent of learning and memory deficits, as determined by Morris water maze (MWM), in aged mice. Enhancing GABA signaling by treating aged apoE4-KI mice with the $\mathrm{GABA}_{\mathrm{A}}$ receptor potentiator pentobarbital (PB) for 4 weeks before and during MWM rescued the learning and memory deficits. Here, we report that withdrawal of PB treatment for 2 weeks before MWM abolished the rescue in aged apoE4-KI mice, suggesting the importance of continuously enhancing GABA signaling in the rescue. However, treating apoE4-KI mice during middle adulthood (9-11 months of age) with PB for 6 weeks prevented age-dependent hilar GABAergic interneuron decline and learning and memory deficits, when examined at 16 month of age. These data imply that increasing inhibitory tone after substantial GABAergic interneuron loss may be an effective symptomatic, but not a disease-modifying, treatment for $\mathrm{AD}$ related to apoE4, whereas a similar intervention before substantial interneuron loss could be a disease-modifying therapeutic.
\end{abstract}

Key words: Alzheimer's disease; apoE; inhibitory neuron; learning and memory; middle adulthood; pentobarbital

Significance Statement

We previously reported that female apoE4-KI mice had an age-dependent decline in hilar GABAergic interneurons that correlated with the extent of cognitive deficits in aged mice. The current study demonstrates that enhancing GABA signaling by treating aged apoE4-KI mice with a $\mathrm{GABA}_{\mathrm{A}}$ receptor potentiator pentobarbital $(\mathrm{PB})$ before and during behavioral tests rescued the cognitive deficits; but withdrawal of $\mathrm{PB}$ treatment for 2 weeks before the tests abolished the rescue, suggesting the importance of continuously enhancing GABA signaling. However, treating apoE4-KI mice during middle adulthood with PB for a short period of time prevented age-dependent hilar GABAergic interneuron decline and cognitive deficits late in life, suggesting early intervention by enhancing GABA signaling as a potential strategy to prevent AD related to apoE4.

\section{Introduction}

Apolipoprotein (apo) E4 is the strongest genetic risk factor for Alzheimer's disease $(\mathrm{AD})$ - the fastest-growing neurodegenerative disease facing mankind (Corder et al., 1993; Farrer et al.,

Received Oct. 19, 2015; revised Jan. 12, 2016; accepted Jan. 18, 2016.

Author contributions: L.M.T. and Y.H. designed research; L.M.T., S.Y.Y., Y.A.-Z., A.Y., V.L., and H.L. performed research; L.M.T., S.Y.Y., Y.A.-Z., A.Y., V.L., H.L., and Y.H. analyzed data; L.M.T. and Y.H. wrote the paper.

This work was supported in part by Grants AG048017, AG047655, NS065780, and RR018928 from the National Institutes of Health, the S.D. Bechtel Jr Foundation, and the Hellman Foundation. We thank Stephen Ordway, Gary Howard, and Philip Nova for editorial assistance.

The authors declare no competing financial interests.

Correspondence should be addressed to Dr Yadong Huang, Gladstone Institute of Neurological Disease, University of California, 1650 0wens Street, San Francisco, CA 94158. E-mail: yadong.huang@gladstone.ucsf.edu.

DOI:10.1523/JNEUROSCI.3815-15.2016

Copyright $\odot 2016$ the authors $\quad 0270-6474 / 16 / 362316-07 \$ 15.00 / 0$
1997; Genin et al., 2011; Huang and Mucke, 2012). In humans, apoE4 exerts increasingly detrimental effects with advanced age and apoE4 homozygosity, especially in females (Farrer et al., 1997; Damoiseaux et al., 2012; Leung et al., 2012). Likewise, in aging female mice with the human APOE4 gene knocked into the mouse apoE locus (apoE4-KI), GABAergic interneurons are progressively impaired and lost in the hilus of the dentate gyrus, likely causing network imbalance and hippocampal hyperactivity, which leads to the observed impairments in spatial learning and memory (Li et al., 2009; Andrews-Zwilling et al., 2010; Leung et al., 2012). Similar network abnormalities and dentate hyperactivity are seen in aging humans with mild cognitive impairment, early stage $\mathrm{AD}$, and cognitively normal apoE4 carriers (Filippini et al., 2009; Dennis et al., 2010). In mouse studies, this population of dentate gyrus interneurons is particularly vulnerable to apoE4, 
especially when produced inside the interneurons themselves (Knoferle et al., 2014). In females (where the effect is more pronounced), regardless of genotype, the number of these cells declines throughout life, but does so in a substantially accelerated manner in mice with apoE4 (Andrews-Zwilling et al., 2010; Leung et al., 2012). A particular subset of the interneuron population expresses the neuropeptide somatostatin (SST), and the decline in SST interneurons most strongly correlates with learning and memory impairments in apoE4-KI mice (Andrews-Zwilling et al., 2010; Leung et al., 2012). Decreases in SST have also been associated with AD in humans (Davies et al., 1980), particularly so among apoE4 carriers (Grouselle et al., 1998).

Previous studies have shown that pentobarbital (PB), a $\mathrm{GABA}_{\mathrm{A}}$ receptor potentiator, pharmacologically increases inhibitory tone and can acutely rescue the deficits of adult neurogenesis in the subgranular zone of the hippocampus and behavioral phenotypes, including learning and memory, in apoE4-KI mice (Li et al., 2009; Andrews-Zwilling et al., 2010). This suggests that rebalancing the GABAergic signaling and inhibitory tone may be the key to rescue the behavioral phenotypes of apoE4-KI mice. $\mathrm{PB}$ is a short-acting $\mathrm{GABA}_{\mathrm{A}}$ receptor potentiator that works by increasing the duration of receptor activation in the presence of GABA, rather than increasing the frequency of activation as with benzodiazepines (Tozuka et al., 2005; Andrews-Zwilling et al., 2010). When aged female apoE4-KI mice are treated with $\mathrm{PB}$ before and during behavioral assessment, learning and memory impairments are rescued (Andrews-Zwilling et al., 2010). However, in the PB-treated apoE4-KI mice, the numbers of SST interneurons remain as low as in the untreated group (AndrewsZwilling et al., 2010), suggesting that functionally enhancing GABA signaling, rather than protecting SST interneuron loss, is responsible for the rescue. We thus hypothesize that in the weeks following the discontinuation of $\mathrm{PB}$ treatment in aged female apoE4-KI mice, in which SST interneuron loss has been substantial, inhibitory signaling as well as learning and memory will return to the impaired baseline levels. We further hypothesize that one strategy to prevent or delay the onset of the behavioral deficits would be to alter the course of interneuron decline at a young age, before SST interneuron loss is substantial enough to cause learning and memory impairments, by treating apoE4-KI mice with PB earlier in life.

We tested these hypotheses in the current study and demonstrate that withdrawal of $\mathrm{PB}$ treatment for 2 weeks in aged apoE4-KI mice before Morris water maze (MWM) test abolished the rescue, confirming the importance of continuously enhancing GABA signaling in the rescue of aged mice. However, treating apoE4-KI mice at a younger age ( 9 months) with PB for 6 weeks prevented learning and memory deficits and ameliorated agedependent hilar GABAergic interneuron decline at an older age (16 months). Thus, early PB treatment prevents late hilar interneuron decline and learning and memory deficits in apoE4-KI mice.

\section{Materials and Methods}

Animals. All protocols and procedures followed the guidelines of the Laboratory Animal Resource Center at the University of California, San Francisco (UCSF). Experimental and control animals had identical housing conditions from birth through euthanasia ( $12 \mathrm{~h}$ light/dark cycle, housed 5/cage, PicoLab Rodent Diet 20). Female apoE3-KI and apoE4-KI homozygous mice on a C57BL/6 background strain (Taconic; Hamanaka et al., 2000) were born and aged under normal conditions at the Gladstone Institutes/UCSF animal facility.
PB Injections. PB (Sigma-Aldrich) was prepared in $0.9 \%$ sterile saline at $5 \mathrm{mg} / \mathrm{ml}$. Mice were administered $20 \mathrm{mg} / \mathrm{kg}$ by intraperitoneal injection for $28 \mathrm{~d}$ ( 4 weeks), 2 weeks before (every morning) and during (every afternoon) behavioral tests (Andrews-Zwilling et al., 2010) or $50 \mathrm{mg} / \mathrm{kg}$ for $42 \mathrm{~d}$ (6 weeks) and discontinued for 2 weeks or 5 months before behavioral tests.

Morris water maze test. All mice were singly housed during Morris water maze (MWM) test, which was conducted in a pool $(122 \mathrm{~cm}$ diameter) with room temperature water $\left(22-23^{\circ} \mathrm{C}\right)$ with a 10 - $\mathrm{cm}$-wide square platform submerged $1.5 \mathrm{~cm}$ below the surface of opaque water during hidden trials (Andrews-Zwilling et al., 2010; Leung et al., 2012; Knoferle et al., 2014). Mice were trained to locate the hidden platform over 4 trials per day for 5 hidden days (HD) $1-5$, where HD0 is the first trial on the first day, with a maximum of $60 \mathrm{~s}$ per trial. Each memory trial (probe trial) was conducted for $60 \mathrm{~s}$ in the absence of the platform at 24,72, and $120 \mathrm{~h}$ after the final learning session. Memory was assessed as percentage of time spent in the target quadrant that contained the platform during the learning trials compared with the average of time spent in the nontarget quadrants. For visible trials, a black-white striped mast $(15 \mathrm{~cm}$ high) marked the platform location. The platform location and room arrangement remained constant throughout the assay with the exception of moving the platform during the visible trials. Speed was calculated by distance traveled divided by trial duration. Performance was objectively monitored using EthoVision video-tracking software (Noldus Information Technology).

Immunohistochemistry. Animals were transcardially perfused with $0.9 \%$ saline and both hemi-brains were collected and drop-fixed in $4 \%$ paraformaldehyde (PFA) for $48 \mathrm{~h}$ at $4^{\circ} \mathrm{C}$. After rinsing in PBS, tissues were cryoprotected in $30 \%$ sucrose. The right hemi-brains were then embedded in a $20 \%$ gelatin $/ 30 \%$ sucrose embedding medium, hardened for $48 \mathrm{~h}$ in $4 \%$ PFA at $4^{\circ} \mathrm{C}$, and cryoprotected in $20 \%$ glycerol $/ 2 \%$ dimethyl sulfoxide for $24 \mathrm{~h}$ at $4^{\circ} \mathrm{C}$ before snap-freezing in an isopentante/ dry-ice slurry. The matrix was then sectioned together coronally in blocks of 12 hemi-brains at $30 \mu \mathrm{m}$ with a frozen sliding microtome (Leica) for floating immunohistochemistry. Floating sheets of brains were blocked in normal rabbit serum (Jackson Immuno Research) with citric acid antigen retrieval ( $1 \mathrm{~mm})$, immunostained overnight with goat anti-SST (1:350; Santa Cruz Biotechnology), followed by incubating with biotinylated rabbit anti-goat IgG (1:500, Jackson Immuno Research) for $1 \mathrm{~h}$ at room temperature. Diaminobenzidine (DAB; Sigma-Aldrich) development was done using Vectastain $\mathrm{ABC}$ amplification kit (Vector Laboratories) for 1-5 min (Andrews-Zwilling et al., 2010; Leung et al., 2012; Knoferle et al., 2014).

Image collection and cell quantification. Histologic images of every $10^{\text {th }}$ section $(30 \mu \mathrm{m})$ across the whole hippocampus of a single hemisphere ( $~ 8$ sections) were collected using Biorevo BZ-9000 Keyence digital microscope. Quantification of SST cells was conducted on images of every section of hippocampus (anterior to posterior) using semiautomated Image-based Tool for Counting Nuclei (ITCN) plugin in ImageJ with manual outlining of the dentate gyrus hilar area, which was defined as the polymorphic nuclear region between the inner border of the granule cell layer and an imaginary connection between the ends of both granule cell blades except for the interposed layer of CA3 pyramidal neurons (Leung et al., 2012), and visual confirmation of counted cells by an investigator blind to the experimental groups. Total hilar cell number was obtained by multiplying the raw count by 20 to account for the 1 in 10 sampling of half the brain (Andrews-Zwilling et al., 2010; Leung et al., 2012; Knoferle et al., 2014).

Statistical analysis. All values are expressed as mean \pm SEM. Statistical analyses were performed with Prism v5.0 software (GraphPad). MWM data were analyzed and compared by repeated measures one-way ANOVA and Bonferroni post hoc test for the hidden training days and by one-way ANOVA and Bonferroni post hoc test for the probe trials. Statistical significance of histochemical quantification was assessed by Student's $t$ test. A $p$ value of $<0.05$ was considered to be statistically significant. Statistical values are denoted as follows: ${ }^{\star} p<0.05,{ }^{* *} p<$ $0.01,{ }^{* * *} p<0.001$. 


\section{Results}

PB treatment of aged apoE4-KI mice before and during MWM rescues learning and memory deficits, but not hilar SST interneuron loss

As reported previously (Andrews-Zwilling et al., 2010), when female 15.5-month-old apoE4-KI mice were given daily PB (20 $\mathrm{mg} / \mathrm{kg}$ ) injection starting 2 weeks before and continued during the 2 weeks of MWM test (Fig. 1A) their learning impairment was rescued, compared with saline-treated apoE4-KI mice, to a level similar to that seen in apoE3-KI mice (Fig. 1B). PB-treated apoE4-KI mice also retained a preference for the target quadrant, whereas saline-treated apoE4-KI mice did not, in probe trials when the platform was removed $72 \mathrm{~h}$ (Fig. 1C) and $120 \mathrm{~h}$ (data not shown) after the hidden trials, suggesting the rescue of apoE4-induced memory deficits by PB treatment. PB treatment of age-matched female apoE3-KI mice did not significantly alter their learning and memory performance (Fig. $1 B, C$ ). There were no significant differences in visible platform trials (Fig. $1 B$ ) or swim speed (Fig. 1D) among all groups of mice. After the behavioral test, brains of all mice were collected, sectioned, and immunostained for SST interneurons, as previously reported (Andrews-Zwilling et al., 2010; Leung et al., 2012; Knoferle et al., 2014). Quantitative analyses revealed that the numbers of hilar SST interneurons in PB-treated and saline-treated apoE4-KI mice were similar, both of which were significantly less than those in PB-treated and saline-treated apoE3-KI (Fig. $1 E-I$ ), indicating that 4 week treatment of aged apoE4-KI mice with PB did not rescue SST interneuron loss.

\section{PB treatment of aged apoE4-KI mice does not have lasting effects on learning and memory improvement after treatment withdrawal}

We then took the advantage of $\mathrm{PB}$ as a short-acting $\mathrm{GABA}_{\mathrm{A}}$ receptor potentiator (Tozuka et al., 2005; Andrews-Zwilling et al., 2010) to determine whether the behavioral rescue in aged apoE4-KI mice requires continuous action of $\mathrm{PB}$ on enhancing GABA signaling or can last for a long period of time after the treatment withdrawal. In this cohort, PB treatment was increased in dosage and duration to $50 \mathrm{mg} / \mathrm{kg}$ and $42 \mathrm{~d}$ to maximize any lasting effects that PB might impart. When MWM was assessed 2 weeks after treatment withdrawal (Fig. 2A), PB-treated apoE4-KI mice no longer displayed rescue of learning and memory deficits compared with saline-treated apoE4-KI mice, both of which performed significantly worse than PB-treated and saline-treated apoE3-KI mice (Fig. 2 B, C). Again, there were no significant differences of performance in visible trials (Fig. $2 B$ ) and swim speed among all groups of mice (Fig. 2D). Similar to the other cohort treated at advanced age, no rescue of hilar SST interneuron loss was observed in aged apoE4-KI mice treated with PB relative to their genotype-matched controls that received saline, and both groups of apoE4-KI mice had reduced numbers of SST interneurons relative to both apoE3-KI groups (Fig. 2E-I). Thus, PB treatment of aged apoE4-KI mice does not have lasting effects on learning and memory improvement after treatment withdrawal.

\section{Early PB treatment prevents late hilar SST interneuron loss and learning and memory deficits in apoE4-KI mice}

To determine whether increasing GABA signaling and inhibitory tone has lasting beneficial effects if done before hilar SST interneuron loss is substantial enough to cause behavioral deficits, $\mathrm{PB}$ treatment was administered to younger adults at 9.5 months of age at $50 \mathrm{mg} / \mathrm{kg}$ daily for $42 \mathrm{~d}$ (Fig. $3 A$ ). Strikingly, when evaluated 5 months after the discontinuation of $\mathrm{PB}$ treatment (Fig. 3A), PB-treated apoE4-KI mice had normal learning and memory similar to those of age-matched apoE3-KI controls (Fig. $3 B, C)$. There were no significant differences of performance in visible trials (Fig. $3 B$ ) and swim speed among all groups of mice (Fig. 3D). Importantly, early PB-treated apoE4-KI mice also had increased numbers of hilar SST interneuron, reaching levels similar to those seen in age- and treatment-matched apoE3-KI mice compared with early saline-treated apoE4-KI mice (Fig. 3E-I). Interestingly, the numbers of hilar SST interneurons in early PBtreated apoE4-KI mice, which were measured at 16 months of age, were comparable with those seen in untreated apoE4-KI mice at 6-12 months of age (Andrews-Zwilling et al., 2010; Leung et al., 2012), suggesting that early PB treatment completely prevents the continuous hilar SST interneuron decline late in life, although the treatment began after there was already a significant difference between apoE4-KI and apoE3-KI mice at 6 months of age (Andrews-Zwilling et al., 2010; Leung et al., 2012). Together, these data indicate that early PB treatment prevents both late severe hilar SST interneuron loss, and learning and memory deficits in apoE4-KI mice.

\section{Discussion}

In this study, we demonstrate that enhancing GABA signaling by $\mathrm{PB}$ treatment in aged apoE4-KI mice before and during behavioral tests rescues learning and memory deficits; however, withdrawal of PB treatment for 2 weeks before behavioral tests abolished the rescue. Thus, the rescue requires continuous treatment with PB in aged apoE4-KI mice. Because the treatment has no effect on hilar SST interneuron decline, it suggests that in aged apoE4-KI mice, in which the hilar SST interneuron loss has been substantial, enhancing GABA signaling is an approach of symptomatic, but not disease-modifying, treatment. However, the age-dependent learning and memory deficits and the severe loss of hilar SST interneurons in apoE4-KI mice can be prevented with early PB treatment for 6 weeks during middle adulthood (9-11 months of age). This provides hope that a brief intervention in middle adulthood to boost inhibitory signaling may rebalance inhibition and excitation and reverse the dentate hyperactivity observed in human apoE4 carriers (Filippini et al., 2009; Dennis et al., 2010), and thus delay or prevent the onset of cognitive decline much later in life.

Furthermore, the early-treated-then-aged apoE4-KI mice have higher numbers of SST interneurons in the hilus of dentate gyrus compared with saline-treated controls. This indicates that early treatment with a potentiator of inhibitory signaling can slow down or even prevent severe inhibitory interneuron loss late in life. $\mathrm{Al}$ though the precise mechanism underlying the protection is still a topic of ongoing investigation, we propose the following working model: in aged mice, apoE4 causes SST interneuron loss in the hilus of the dentate gyrus, leading to impaired inhibition (AndrewsZwilling et al., 2010, 2012; Li et al., 2009) and, potentially, excitotoxic injury. This imbalance of inhibition and excitation may contribute to deficits in learning and memory (Andrews-Zwilling et al., 2012). Meanwhile, the overexcitation likely causes more SST interneuron death, initiating a vicious cycle. When aged apoE4-KI mice are treated with the $\mathrm{GABA}_{\mathrm{A}}$ receptor potentiator $\mathrm{PB}$, the excitotoxicity is likely suppressed and inhibition and excitation are rebalanced. This is enough to have a positive effect on improving learning and memory behavior. However, because hilar SST interneuron loss is already substantial in aged apoE4-KI mice, enhancing GABA signaling cannot rescue their loss; thus, withdrawal of $\mathrm{PB}$ treatment abolishes the temporary rescue of learning and memory deficits. Importantly, 

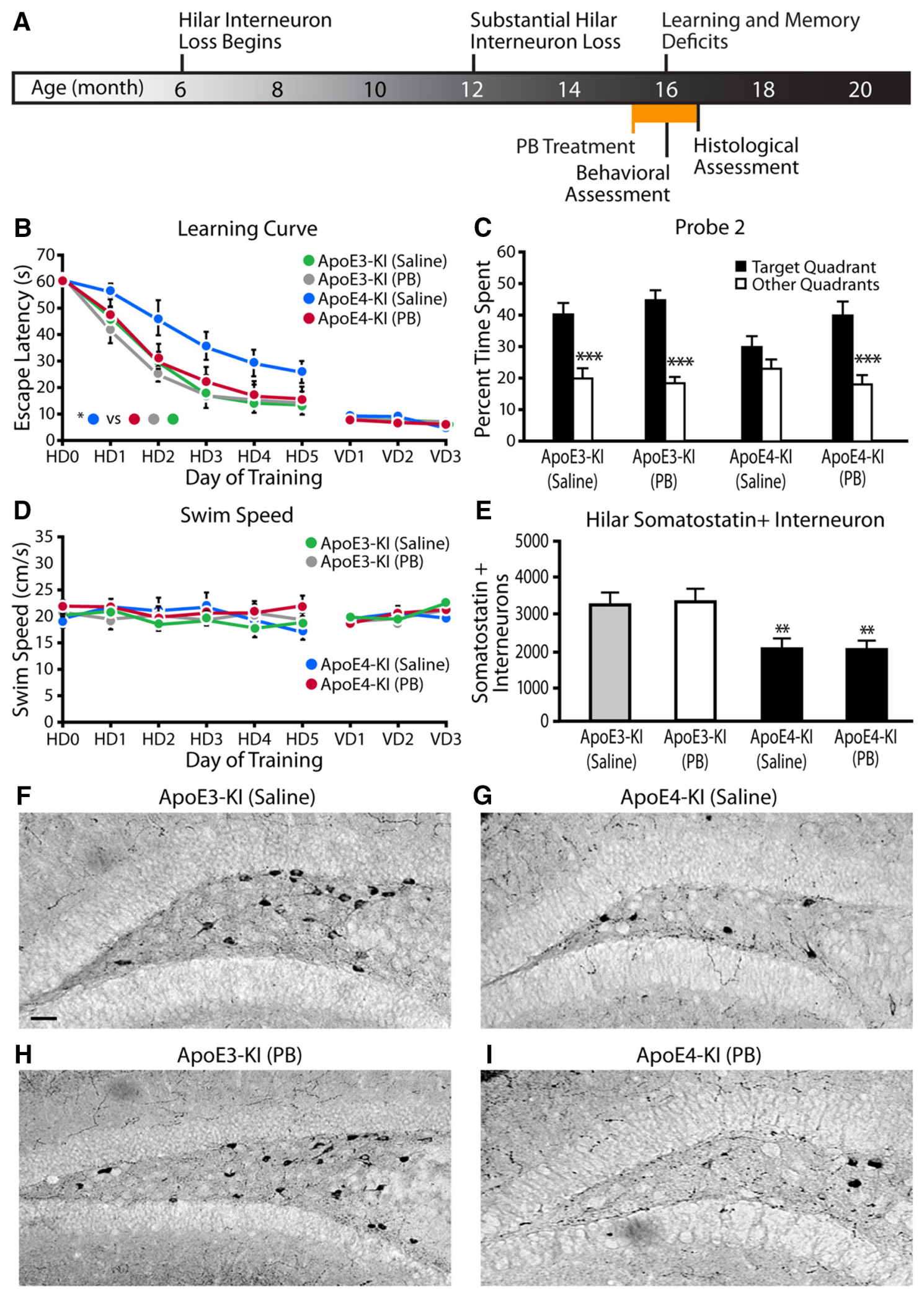

Figure 1. PB treatment of aged apoE4-KI mice before and during MWM test rescued learning and memory deficits, but not hilar SST interneuron loss. $A$, Experimental timeline for 4 weeks of PB treatment before and during behavioral assessment in MWM followed by histological analysis. $\boldsymbol{B}$, Hidden trials of MWM test showed that PB treatment before and during the test rescued learning impairment in aged apoE4-KI mice. There were no significant differences in visible trials among all groups. Points represent averages of daily trials. HD, hidden platform day (2 trials/session, 2 sessions/d); HDO, First trial on HD1; VD, visible platform day (2 trials/session, 2 sessions/d). $y$-axis indicates the time to reach the target platform (escape latency, mean \pm SEM). C, A probe trial performed $72 \mathrm{~h}$ after the last hidden trial showed that PB treatment before and during the test also rescued memory impairment in aged apoE4-KI mice. D, Swim speeds did not differ significantly among all groups. $\boldsymbol{E}$, Quantification of total hilar SST interneurons after behavioral assessments showed no rescue of hilar SST interneuron loss. $\boldsymbol{F}-\boldsymbol{I}$, Representative images of DAB-stained hilar SST interneurons in saline-treated apoE3-KI $(\boldsymbol{F})$, saline-treated apoE4-KI $(\boldsymbol{G})$, PB-treated apoE3-KI $(\boldsymbol{H})$, and PB-treated apoE4-KI $(\boldsymbol{I})$ mice. Values are mean \pm SEM. ${ }^{*} p<0.05,{ }^{* *} p<0.01$, ${ }^{* * *} p<$ 0.001 as determined by repeated-measures two-way ANOVA $(\boldsymbol{B})$ and unpaired Student's $t$ test $(\boldsymbol{C}, \boldsymbol{E})$. Scale bar: (in $\boldsymbol{F}) \boldsymbol{F}-\boldsymbol{I}, 50 \mu \mathrm{m} . n=7-12$ mice per group. 

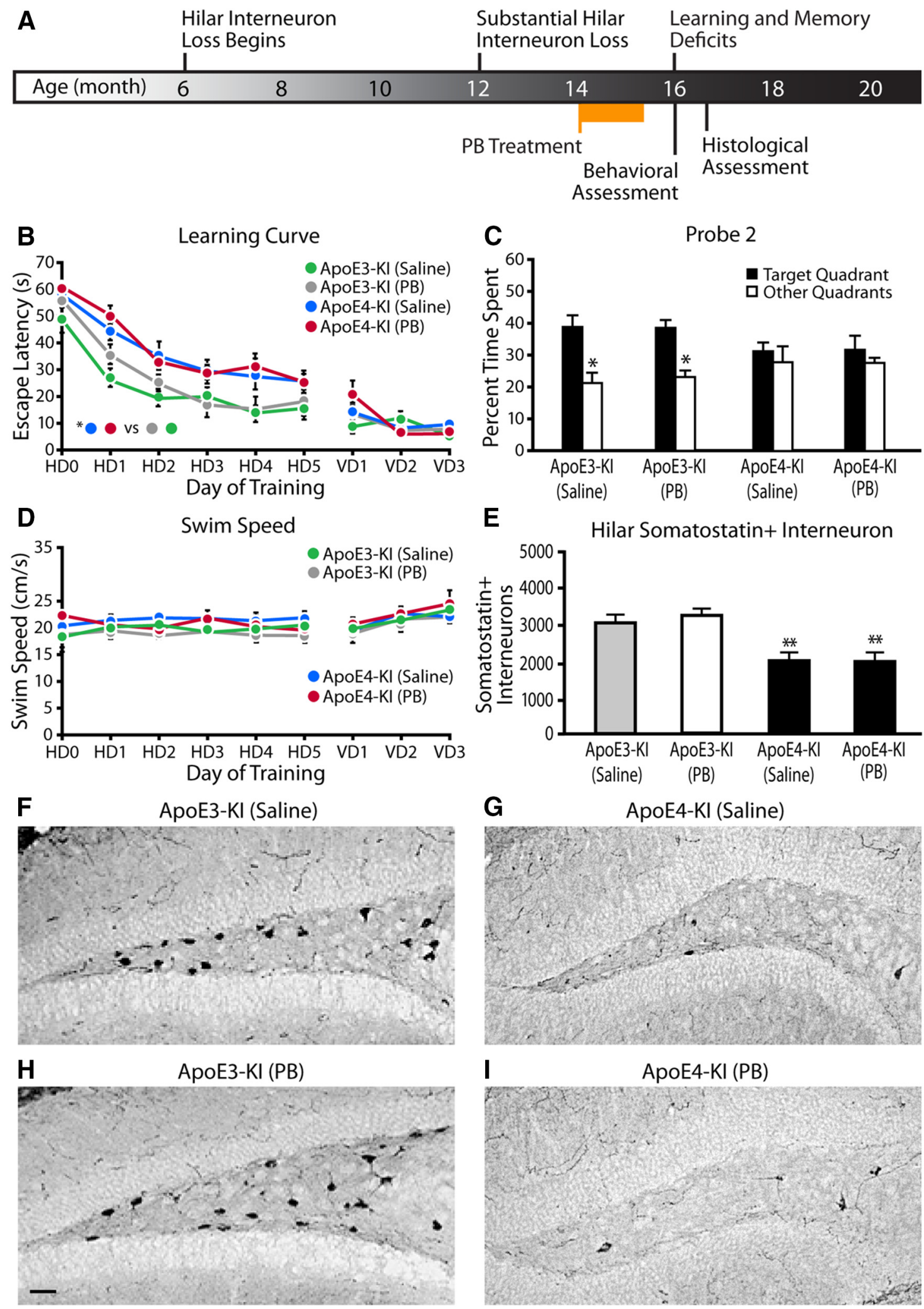

Figure 2. PB treatment of aged apoE4-KI mice did not have lasting effects on learning and memory improvement after treatment withdrawal. $A$, Experimental timeline for 6 weeks of $\mathrm{PB}$ treatment $\mathrm{foll}$ owed by treatment withdrawal for 2 weeks and then behavioral assessment in MWM followed by histologic analysis. $B$, Hidden trials of MWM test showed that withdrawal of PB treatment for 2 weeks before Morris water maze (MWM) test abolished the rescue of learning impairment in aged apoE4-KI mice. There were no significant differences in visible trials among all groups. Points represent averages of daily trials. HD, Hidden platform day (2 trials/session, 2 sessions/d); HDO, first trial on HD1; VD, visible platform day (2 trials/session, 2 sessions/d). $y$-axis indicates time to reach the target platform (escapelatency, mean \pm SEM). C, A probe trial performed $72 \mathrm{~h}$ after the last hidden trial showed that withdrawal of PB treatment for 2 weeks before MWM test abolished the rescue of memory impairment in aged apoE4-KI mice. $D$, Swim speeds did not differ significantly among all groups. $\boldsymbol{E}$, Quantification of total hilar SST interneurons after behavioral assessments showed no rescue of hilar SST interneuron loss. $\boldsymbol{F}$ - I, Representative images of DAB-stained hilar SST interneurons in saline-treated apoE3-KI $(\boldsymbol{F})$, saline-treated apoE4-KI $(\boldsymbol{G})$, PB-treated apoE3-KI $(\boldsymbol{H})$, and PB-treated apoE4-KI $(\boldsymbol{I})$ mice. Values are mean \pm SEM. ${ }^{*} p<0.05,{ }^{* *} p<0.01$ as determined by repeated-measures two-way ANOVA (B) and unpaired Student's t test $(\boldsymbol{C}, \boldsymbol{E})$. Scale bar: (in $\boldsymbol{H}) \boldsymbol{F}-\boldsymbol{I}, 50 \mu \mathrm{m} . n=10-12$ mice per group. 

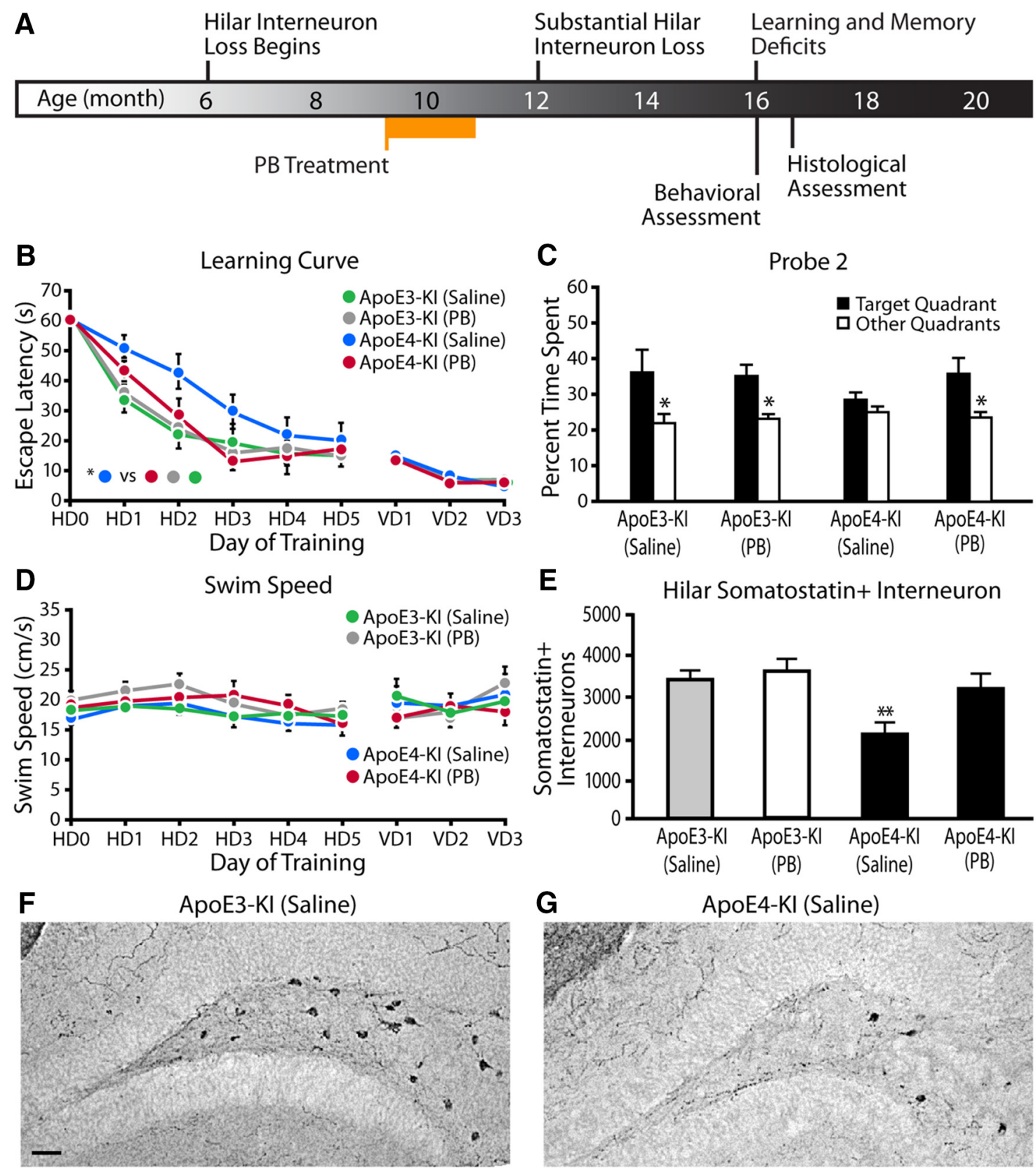

G ApoE4-KI (Saline)
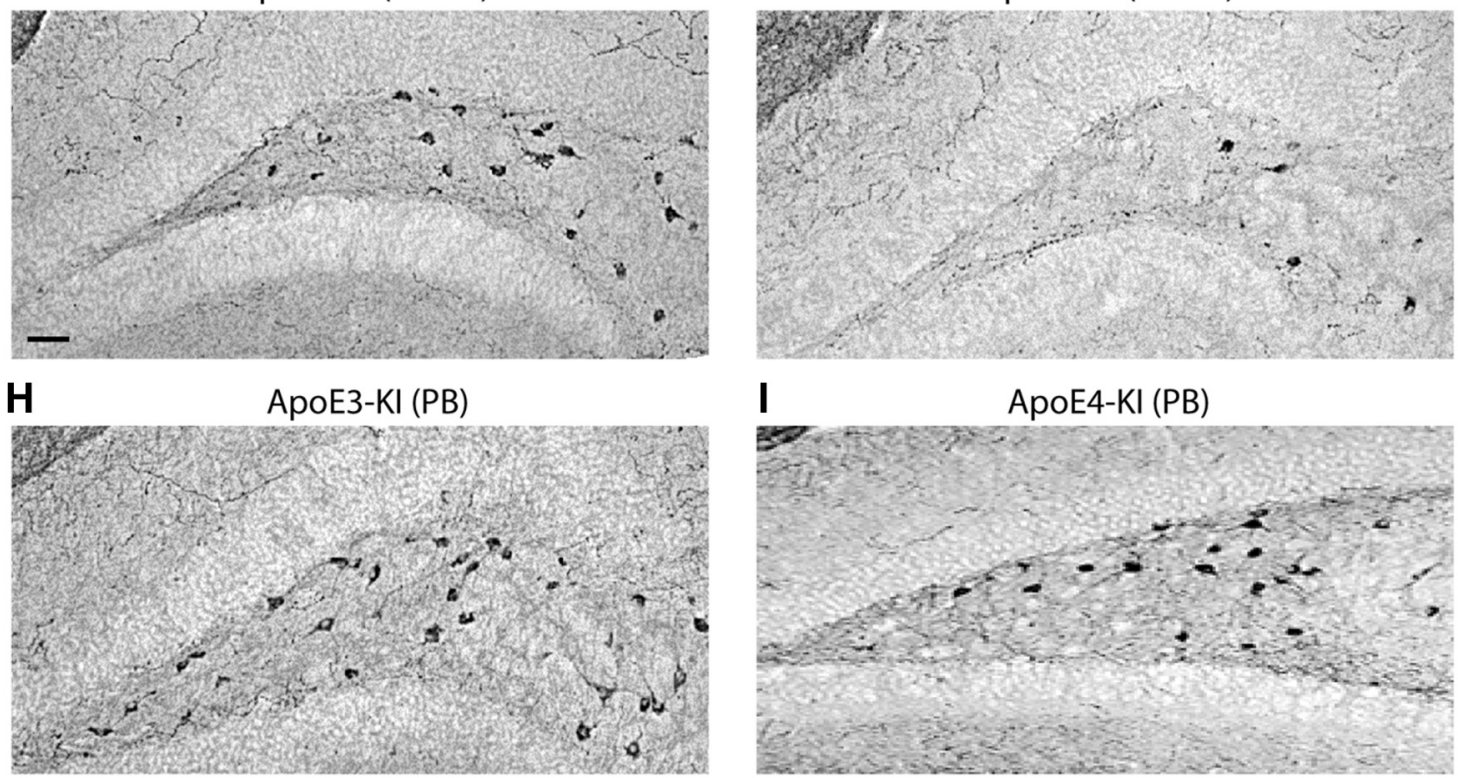

Figure 3. Early PB treatment prevented late hilar SST interneuron loss and learning and memory deficits in apoE4-KI mice. A, Experimental timeline for 6 weeks of PB treatment starting at 9.5 months of age and behavioral assessment at 16 months of age in MWM followed by histologic analysis. $\boldsymbol{B}$, Hidden trials of MWM test showed that early PB treatment prevented late learning deficit in apoE4-KI mice. There were no significant differences in visible trials among all groups. Points represent averages of daily trials. HD, Hidden platform day (2 trials/session, 2 sessions/d); HDO, first trial on HD1; VD, visible platform day (2 trials/session, 2 sessions/d). $y$-axis indicates time to reach the target platform (escape latency, mean \pm SEM). C, A probe trial performed $72 \mathrm{~h}$ after the last hidden trial showed that early PB treatment also prevented late memory deficit in apoE4-KI mice. D, Swim speeds did not differ significantly among all groups. E, Quantification of total hilar SST interneurons after behavioral assessments showed that early PB treatment prevented late hilar SST interneuron loss. $\boldsymbol{F}-\boldsymbol{I}$, Representative images of DAB-stained hilar SST interneurons in saline-treated apoE3-KI $(\boldsymbol{F})$, saline-treated apoE4-KI (G), PB-treated apoE3-KI $(\boldsymbol{H})$, and PB-treated apoE4-KI $(\boldsymbol{I})$ mice. Values are mean \pm SEM. ${ }^{*} p<0.05,{ }^{* *} p<0.01$ as determined by repeated-measures two-way ANOVA (B) and unpaired Student's t test $(\boldsymbol{C}, \boldsymbol{E})$. Scale bar: (in $\boldsymbol{F}) \boldsymbol{F}-\boldsymbol{I}, 50 \mu \mathrm{m} . n=7-11$ mice per group. 
when $\mathrm{PB}$ treatment starts at middle adulthood in apoE4-KI mice, it interrupts the cycle of impaired inhibition and overexcitation, leading to prevention of age-dependent hilar SST interneuron loss and, consequently, preservation of normal learning and memory. Although we did not evaluate in the current study whether early PB treatment completely prevents or merely delays the onset of learning and memory deficits in apoE4-KI mice, even a delay of onset would be a substantial improvement to current treatment options. It is important to mention that $\mathrm{PB}$ may not be an ideal drug for treating $\mathrm{AD}$ patients due to safety issues, such as the sedative side effects; thus, alternative approaches would also need to be explored for increasing inhibitory tone or confining the effects of $\mathrm{PB}$ to the hippocampus. Nonetheless, our data suggest that early intervention to enhance GABA signaling could be a potential strategy to prevent AD related to apoE4.

\section{References}

Andrews-Zwilling Y, Bien-Ly N, Xu Q, Li G, Bernardo A, Yoon SY, Zwilling D, Yan TX, Chen L, Huang Y (2010) Apolipoprotein E4 causes age- and tau-dependent impairment of GABAergic interneurons, leading to learning and memory deficits in mice. J Neurosci 30:13707-13717. CrossRef Medline

Andrews-Zwilling Y, Gillespie AK, Kravitz AV, Nelson AB, Devidze N, Lo I, Yoon SY, Bien-Ly N, Ring K, Zwilling D, Potter GB, Rubenstein JLR, Kreitzer AC, Huang Y (2012) Hilar GABAergic interneuron activity controls spatial learning and memory retrieval. PloS One 7:e40555. CrossRef Medline

Corder EH, Saunders AM, Strittmatter WJ, Schmechel DE, Gaskell PC, Small GW, Roses AD, Haines JL, Pericak-Vance MA (1993) Gene dose of apolipoprotein E type 4 allele and the risk of Alzheimer's disease in late onset families. Science 261:921-923. CrossRef Medline

Damoiseaux JS, Seeley WW, Zhou J, Shirer WR, Coppola G, Karydas A, Rosen HJ, Miller BL, Kramer JH, Greicius MD (2012) Gender modulates the APOE $\varepsilon 4$ effect in healthy older adults: convergent evidence from functional brain connectivity and spinal fluid tau levels. J Neurosci 32:8254-8262. CrossRef Medline

Davies P, Katzman R, Terry RD (1980) Reduced somatostatin-like immunoreactivity in cerebral cortex from cases of Alzheimer disease and Alzheimer senile dementia. Nature 288:279-280. CrossRef Medline

Dennis NA, Browndyke JN, Stokes J, Need A, Burke JR, Welsh-Bohmer KA,
Cabeza R (2010) Temporal lobe functional activity and connectivity in young adult APOE $\varepsilon 4$ carriers. Alzheimers Dement 6:303-311. CrossRef Medline

Farrer LA, Cupples LA, Haines JL, Hyman B, Kukull WA, Mayeux R, Myers RH, Pericak-Vance MA, Risch N, van Duijn CM (1997) Effects of age, sex, and ethnicity on the association between apolipoprotein E genotype and Alzheimer disease: a meta-analysis. JAMA 278:1349-1356. CrossRef Medline

Filippini N, MacIntosh BJ, Hough MG, Goodwin GM, Frisoni GB, Smith SM, Matthews PM, Beckmann CF, Mackay CE (2009) Distinct patterns of brain activity in young carriers of the APOE- $\varepsilon 4$ allele. Proc Natl Acad Sci U S A 106:7209-7214. CrossRef Medline

Genin E, Hannequin D, Wallon D, Sleegers K, Hiltunen M, Combarros O, Bullido MJ, Engelborghs S, De Deyn P, Berr C, Pasquier F, Dubois B, Tognoni G, Fiévet N, Brouwers N, Bettens K, Arosio B, Coto E, Del Zompo M, Mateo I, et al. (2011) APOE and Alzheimer disease: a major gene with semidominant inheritance. Mol Psychiatry 16:903-907. CrossRef Medline

Grouselle D, Winsky-Sommerer R, David JP, Delacourte A, Dournaud P, Epelbaum J (1998) Loss of somatostatin-like immunoreactivity in the frontal cortex of Alzheimer patients carrying the apolipoprotein epsilon 4 allele. Neurosci Lett 255:21-24. CrossRef Medline

Hamanaka H, Katoh-Fukui Y, Suzuki K, Kobayashi M, Suzuki R, Motegi Y, Nakahara Y, Takeshita A, Kawai M, Ishiguro K, Yokoyama M, Fujita SC (2000) Altered cholesterol metabolism in human apolipoprotein E4 knock-in mice. Hum Mol Genet 9:353-361. CrossRef Medline

Huang Y, Mucke L (2012) Alzheimer mechanisms and therapeutic strategies. Cell 148:1204-1222. CrossRef Medline

Knoferle J, Yoon SY, Walker D, Leung L, Gillespie AK, Tong LM, Bien-Ly N, Huang Y (2014) Apolipoprotein E4 produced in GABAergic interneurons causes learning and memory deficits in mice. J Neurosci 34:14069-14078. CrossRef Medline

Leung L, Andrews-Zwilling Y, Yoon SY, Jain S, Ring K, Dai J, Wang MM, Tong L, Walker D, Huang Y (2012) Apolipoprotein E4 causes age- and sex-dependent impairments of hilar GABAergic interneurons and learning and memory deficits in mice. PLoS One 7:e53569. CrossRef Medline

Li G, Bien-Ly N, Andrews-Zwilling Y, Xu Q, Bernardo A, Ring K, Halabisky B, Deng C, Mahley RW, Huang Y (2009) GABAergic interneuron dysfunction impairs hippocampal neurogenesis in adult apolipoprotein E4 knockin mice. Cell Stem Cell 5:634-645. CrossRef Medline

Tozuka Y, Fukuda S, Namba T, Seki T, Hisatsune T (2005) GABAergic excitation promotes neuronal differentiation in adult hippocampal progenitor cells. Neuron 47:803-815. CrossRef Medline 